\title{
Bilateral testicular infarction as a complication of acute cardiovascular diseases
}

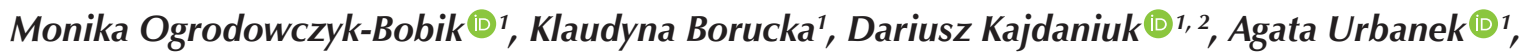 \\ Szymon Janyga ${ }^{1}$, Franciszek Mazur ${ }^{1}$, Tomasz Miś1, Bogdan Marek (1) 1, \\ ${ }^{1}$ Department of Endocrinology and Metabolic Diseases, Regional Specialist Hospital No. 3, Rybnik, Poland \\ ${ }^{2}$ Department of Pathophysiology and Endocrinology, Faculty of Medical Sciences in Zabrze, Medical University of Silesia, Katowice, \\ Poland
}

Key words: hypergonadotropic hypogonadism; testicular infarction; cardiogenic shock

The paper presents the case of a 59-year-old man with hypergonadotropic hypogonadism in the course of bilateral testicular infarction, which was most likely caused by a drop in system blood pressure in the course of cardiogenic shock complicating myocardial infarction or thrombosis of the iliac arteries, which appeared on the second day after coronary artery bypass surgery.

A 59-year-old man was admitted to the endocrinology department for the diagnosis of hypogonadism. The patient reported a year of fatigue, decreased libido, erectile dysfunction, and a progressive weight gain of $6 \mathrm{~kg}$ over a period of 12 months. He was treated for ischaemic heart disease, chronic heart failure, hypercholesterolaemia, generalized atherosclerosis, and prostatic hyperplasia. At the beginning of 2020, he had an no ST elevation myocardial infarction (NSTEMI) accompanied by cardiogenic shock, treated urgently with aortic-coronary bypass surgery and plastic surgery of the mitral valve due to its high regurgitation. On the second day after surgery, bilateral thrombosis of the iliac and femoral arteries occurred, which was treated with embolectomy. On admission to the endocrinology department, the following deviations from the normal state were noted in a physical examination: overweight [body mass index $(\mathrm{BMI})=29.5$ ], thinning hair on the face, chest, axillary and pubic area, bilateral gynaecomastia, in the scrotum testes were of normal size, with reduced cohesiveness. The performed diagnostics allowed for the diagnosis of hypergonadotropic hypogonadism.

Other parameters were as follows: luteinizing hormone (LH) - $20.39 \mathrm{mIU} / \mathrm{mL}$ (0.57-12.07), follicle-stimulating hormone (FSH) - $45.85 \mathrm{mIU} / \mathrm{mL}$ (0.95-11.95), testosterone - $0.81 \mathrm{ng} / \mathrm{mL}$ (1.43-9.23), sex hormone binding globulin (SHBG) - $39.74 \mathrm{nmol} / \mathrm{L}$ (13.5-71.4), free androgen index $(\mathrm{FAI})-7 \%$, oestradiol $<11 \mathrm{pg} / \mathrm{mL}$.

There were no abnormalities in the functioning of the pituitary, thyroid, and adrenal glands. In addition, quite low dehydroepiandrosterone (DHEAS) values of 80.4 $\mu \mathrm{g} / \mathrm{dL}(<295 \mu \mathrm{g} / \mathrm{dL})$ suggest that the testicles did not release DHEAS. The elevated titre of anti-thyroglobulin antibodies $(74.83 \mathrm{IU} / \mathrm{mL}$ ) had no effect on thyroid function. In basic laboratory tests, apart from a slight low density lipoprotein (LDL) hypercholesterolaemia of $2.86 \mathrm{mmol} / \mathrm{L}(<2.6)$, no abnormalities were found. Also, the values of prostate-specific antigen (PSA) and gonadal markers such as beta-human chorionic gonadotrophin (bhCG), lactate dehydrogenase (LDH), and alpha fetal protein (AFP) and the oral glucose tolerance test were normal.

An ultrasound examination of the thyroid gland revealed the features of autoimmune inflammation. Breast ultrasound showed small hypoechogenic areas in the sub-papillary regions, $7 \times 9 \mathrm{~mm}$ in the right breast and $12 \times 11 \mathrm{~mm}$ in the left breast, corresponding to gynaecomastia. In an ultrasound of the scrotum, both gonads were without vascular flow, with a hypoechogenic zone predominating in the central part corresponding to necrosis, and a normoechogenic layer of the normal testis on the perimeter of 1-2 mm. The image was typical of an infarction of both testes (Fig. 1).

In order to rule out possible ischaemia of other endocrine glands (currently without clinical implications), MRI of the pituitary gland was performed, in which no abnormalities were found. Due to the irreversible nature of the lesions - complete necrosis of 


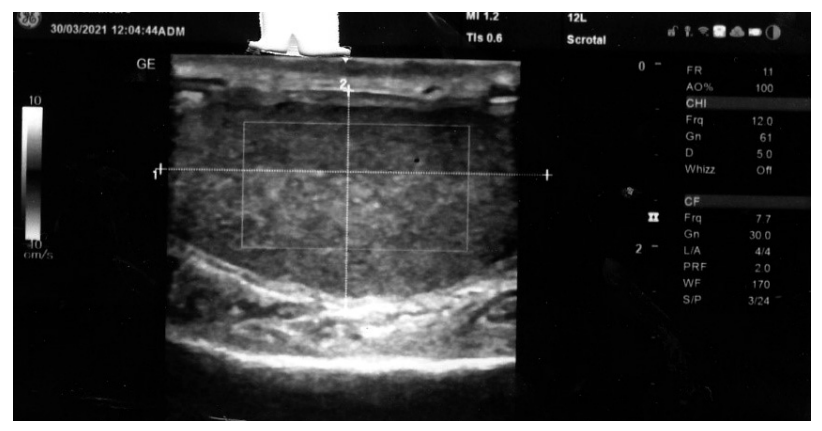

Figure 1. An ultrasound examination of the right testicle

both testes - replacement therapy remains the treatment of choice. Transdermal testosterone in 2 doses a day was considered optimal. Endocrine check-up was recommended to monitor testosterone, oestradiol, and haematocrit levels, and urological check-up to evaluate the prostate. The effect of testosterone treatment on the course/reduction of gynaecomastia should be assessed in a follow-up breast ultrasound performed after 3 months of therapy. Considering the cardiovascular risk, currently no anti-oestrogen drugs have been recommended due to their potential pro-thrombotic effects.

The most probable cause of testicular damage seems to be their ischaemia, either in the course of cardiogenic shock or postoperative thrombosis of the femoral and iliac arteries. Lerisch syndrome seems unlikely due to the pulse present in the peripheral arteries. Due to the long history, the changes in the testicles are irreversible and qualify only for symptomatic treatment.
Testicular ischaemia is most often the result of torsion of the testicle stalk. Less common causes include neoplasms, inflammatory processes, pelvic surgery, malformations, sickle cell anaemia, and vasculitis [1-3]. After 4-8 hours of reduced blood flow to the testes, irreversible changes occur as a result of testicular cell apoptosis. The vasculature of the testes consists of testicular arteries (branches of the aorta), arteries to the ductus deferens (branches of the internal iliac artery), and the cremasteric artery (branch of the inferior epigastric artery - the branch of the external iliac artery), hence the possibility of testicular ischaemia as a result of thromboembolic changes in the iliac arteries. Cardiogenic shock, due to circulatory centralization and reduced peripheral perfusion, may lead to testicular tissue necrosis. More often, however, it is chronic cardiovascular diseases that can cause both hypo- and hypergonadotropic hypogonadism. On the other hand, low blood testosterone concentration is a risk factor for cardiovascular diseases, and testosterone replacement therapy in selected cases may reduce this risk $[4,5]$.

\section{References}

1. Parkin CJ, Kam J, Yuminaga Y, et al. Segmental testicular infarction, a rare complication of epididymo-orchitis. Urol Case Rep. 2020; 32: 101246, doi: 10.1016/j.eucr.2020.101246, indexed in Pubmed: 32489887.

2. Ates E, Kazici HG, Amasyali AS. A rare complication of inguinal hernia repair: Total testicular ischemia and necrosis. Arch Ital Urol Androl. 2019; 91(1): 46-48, doi: 10.4081/aiua.2019.1.46, indexed in Pubmed: 30932429.

3. Clarke K, Campbell C, Omoyinmi E, et al. Testicular ischemia in deficiency of adenosine deaminase 2 (DADA2). Pediatr Rheumatol Online J. 2019; 17(1): 39, doi: 10.1186/s12969-019-0334-5, indexed in Pubmed: 31291964.

4. Kloner RA, Carson C, Dobs A, et al. Testosterone and Cardiovascular Disease. J Am Coll Cardiol. 2016; 67(5): 545-557, doi: 10.1016/j. jacc.2015.12.005, indexed in Pubmed: 26846952.

5. Kirby M, Hackett G, Ramachandran S. Testosterone and the Heart. Eur Cardiol. 2019; 14(2): 103-110, doi: 10.15420/ecr.2019.13.1, indexed in Pubmed: 31360231. 